\title{
Profile and validation of dysregulated long non-coding RNAs and mRNAs in ovarian cancer
}

\author{
YAN-MING LU, YUE WANG, SHI-QI LIU, MENG-YA ZHOU and YA-RU GUO \\ Department of Gynecology and Obstetrics, The Affiliated Shengjing Hospital, \\ China Medical University, Shenyang, Liaoning 110003, P.R. China
}

Received January 20, 2018; Accepted July 19, 2018

DOI: $10.3892 /$ or.2018.6654

\begin{abstract}
The dysregulation of long non-coding RNAs (lncRNAs) is associated with cancer development. The present study profiled differentially expressed lncRNAs in ovarian cancer (OC) versus normal ovarian tissues (NT) and investigated their potential functions in gene expression. OC tissues from 30 patients and NT specimens from 20 non-tumor patients were collected, and 5 cases of tumor and NT were subjected to lncRNA and mRNA microarray analysis. Gene Ontology (GO) and Kyoto Encyclopedia of Genes and Genomes pathway analyses were performed. Reverse transcription-quantitative polymerase chain reaction (RT-qPCR) was used to verify microarray data in all 30 cases. There were 2,870 differentially expressed lncRNAs (795 upregulated and 2,075 downregulated) and 2,658 differentially expressed mRNAs (1,014 upregulated and 1,644 downregulated) in OC. A total of 4 upregulated and 4 downregulated lncRNAs were validated using RT-qPCR. The data demonstrated that, with the exception of ENST00000453838 and ENST00000505048, the lncRNAs were consistent with the microarray data. Another differentially expressed lncRNA (BC041954) was assessed using independent tissue samples, and results further supported the microarray data. Moreover, GO analysis showed that the upregulated genes were involved in the 'development of the cell anatomical structure' (GO: 0048856; $\mathrm{P}=5.46 \times 10^{-6}$ ), 'embryo and system development' and 'multicellular organismal development' in biological processes. By contrast, the downregulated genes were involved in 'gene expression' (GO: 0010476; $\left.\mathrm{P}=1.81 \times 10^{-6}\right)$, 'nitrogen compound metabolic process', 'kidney development' and the 'cellular nitrogen compound metabolic process'. These differentially expressed lncRNAs could be classified into four classes, namely, the enhancer
\end{abstract}

Correspondence to: Dr Yan-Ming Lu, Department of Gynecology and Obstetrics, The Affiliated Shengjing Hospital, China Medical University, 36 Sanhao Street, Heping, Shenyang, Liaoning 110003, P.R. China

E-mail: luyanming555@163.com

Key words: long non-coding RNA, microarray, ovarian cancer, reverse transcription-quantitative polymerase chain reaction
lncRNA nearby coding gene, HOX cluster, long-intergenic non-coding RNAs (lincRNAs) nearby coding gene and Rinn lincRNAs. Coding-non-coding gene co-expression network analysis showed the interregulation of lncRNAs and mRNAs in OC development. In conclusion, dysregulated lncRNA and mRNA expression could promote OC development. Further study may validate a number as OC markers and provide novel insights into ovarian cancer biology.

\section{Introduction}

Ovarian cancer (OC) remains a significant health problem in women and is the second most common gynecological malignancy in the female reproductive system. OC is the ranked first in terms of causing mortality among all the gynecological tumors and poses a serious threat to human life, although the incidence of OC is only third after cervical and endometrial cancer $(1,2)$. Globally, in 2012, $>238,700$ women were diagnosed with OC and an estimated 151,900 patients succumbed to the disease (2). Histologically, epithelial ovarian carcinoma (EOC) contributes to $\sim 90 \%$ of all OC cases and is diagnosed at advanced stages (3). Despite advancements in treatment options and molecular studies, the 5-year survival rate for advanced EOC patients has not been improved and has never exceeded $30 \%$ over the past several decades, leading to a poor EOC prognosis (4). An early diagnosis is the key to reducing overall mortality and increasing survival. However, the molecular mechanisms of action behind the aggressive clinical behaviors of OC are not fully understood, and OC pathogenesis is a complex biological process that involves gene transcriptional regulation and epigenetic regulation $(5,6)$. Thus, a better understanding of the molecular alterations and gene regulations involved will lead to improvements in OC prevention, early diagnosis and treatment options.

Human genome sequences show that only $\sim 2 \%$ of the human genome codes for proteins and that the majority of the genome codes for non-coding RNAs (ncRNAs) (7); however, the functions of these ncRNAs remain to be determined. Accumulating evidence has shown that these ncRNAs could regulate gene expression at the transcriptional and post-transcriptional levels and that they thus serve a critical role in the majority of biological processes (8). ncRNAs can be further divided into microRNAs (miRNAs/miRs) and long 
ncRNAs (lncRNAs) according to their sequence length $(9,10)$. To date, miRNAs have been well studied and have demonstrated multiple functions and aberrant expression in a number of human cancer types, including OC; for example, the expression of miR-34b, miR-200c and miR-141 was found to be altered and associated with $\mathrm{OC}$ development in previous studies $(11,12)$.

Molecules of IncRNAs are $>200$ nucleotides in length and are typically divided into exonic, intronic, overlapping and intergenic according to their genomic localization and in relation to the nearby protein-coding transcripts (13). Initially, due to their poor evolutionary conservation relative to the protein coding regions of the genome, they were considered as transcriptional noise; however, they are currently recognized as novel regulators of gene expression, as well as the altered expression and function involved in human diseases such as cancer (14). A number of high-throughput profiling and RNA sequencing studies have identified a variety of differentially expressed lncRNAs in different cancer types, which is a hallmark feature in human cancer $(15,16)$. Dysregulated expression of lncRNAs was shown to contribute to cancer development, as the IncRNAs can regulate various cell processes through epigenetic regulation (5), microRNA silencing (6), DNA damage and cell cycle control $(8,9)$. Moreover, lncRNAs may also be a potential strategy as therapeutic targets for human diseases, such as cancer, or as biomarkers for the early diagnosis and prediction of prognosis for human cancer (15). Notably, recent studies showed aberrant expression of IncRNAs in breast (17), hepatocellular (18), ovarian (19), and colorectal (20) cancer.

In the present study, dysregulated IncRNA expression in OC versus normal ovarian tissues (NT) was profiled using microarray analysis. Bioinformatic analyses [such as Gene Ontology, Kyoto Encyclopedia of Genes and Genomes (KEGG) pathway and network analyses] were then performed to investigate the potential functions of these target genes of the dysregulated lncRNAs. Microarray data was also validated using reverse transcription-quantitative polymerase chain reaction (RT-qPCR) in another set of tissue samples.

\section{Materials and methods}

Study population. This study collected tissue specimens from 30 OC patients and 20 non-tumor patients from Shengjing Hospital, China Medical University (Shenyang, Liaoning, China) between January 2013 and December 2015. All OC patients were histologically diagnosed and classified according to the World Health Organization criteria $(21,22)$ and had not received any preoperative chemoradiation. OC samples were collected from women who underwent cancer resection. NT samples were collected from women of the same age group who underwent hysterectomy for non-malignant conditions. This study was approved by the Ethics Committee of Shengjing Hospital, China Medical University, and each patient provided written informed consent for the collection of tissue samples that were obtained during surgery. In the study, 5 OC and 5 NT samples were randomly selected and used for microarray analysis, and the remaining $25 \mathrm{OC}$ and $15 \mathrm{NT}$ samples were used for validation purposes. The clinicopathological data for these patients are shown in Table I.
RNA isolation. Tissue samples were collected, and were snap-frozen in liquid nitrogen immediately after resection. Hematoxylin and eosin staining was first performed on the tissue samples to confirm their histology, and then total RNA was isolated using TRIzol reagent (Invitrogen; Thermo Fisher Scientific, Inc., Waltham, MA, USA) according to the manufacturer's protocols. The RNA concentration and quality were then assessed using NanoDrop technology (Thermo Fisher Scientific, Inc.) and 1\% agarose gel electrophoresis, respectively. Optical density 260/280 absorbance ratios were between 1.8 and 2.0 for all the samples.

Microarray analysis. Human lncRNA microarray v2.0 was obtained from Arraystar Co. (Rockville, MD, USA) for global profiling of human lncRNAs and protein-coding transcripts. This microarray contains 33,045 lncRNAs and 30,215 mRNA transcripts. For microarray analysis, the Agilent Array platform (Agilent Technologies, Inc., Santa Clara, CA, USA) was utilized to prepare the samples and microarray hybridization was performed following the manufacturer's protocols with minor modifications, which was all conducted by KangChen Biotech Company (Shanghai, China). Briefly, mRNA was purified from the total RNA samples using the mRNA-ONLY ${ }^{\mathrm{TM}}$ Eukaryotic mRNA Isolation kit (Epicentre; Illumina, Inc., San Diego, CA, USA). Next, these mRNA samples were amplified and transcribed into fluorescent cRNA probes without 3'-bias utilization of a randomly priming method. Furthermore, these labeled cRNA probes were then purified with the RNeasy Mini kit (Qiagen, Inc., Valencia, CA, USA). The concentration and activity of the cRNA probes, expressed as $\mathrm{pM} \mathrm{Cy} 3 / \mu \mathrm{g}$ cRNA, were estimated with the NanoDrop ND-1000 (Thermo Fisher Scientific, Inc.). Next, $1 \mu \mathrm{g}$ of the labeled cRNA probe was fragmented and then diluted with $25 \mu \mathrm{l} 2 \mathrm{X}$ GE hybridization buffer (from the Agilent Array platform). For array hybridization, hybridization solution $(50 \mu \mathrm{l}$ each) was added into the gasket slides; they were then incubated at $65^{\circ} \mathrm{C}$ for $17 \mathrm{~h}$. The next day, the arrays were washed with different buffer (from the Agilent Array platform) and scanned with the Agilent DNA microarray scanner (model no. G2505C; Agilent Technologies, Inc.).

Data analysis. The Agilent Feature Extraction software (version 11.0.1.1; Agilent Technologies, Inc.) and GeneSpring GX v12.0 software package (Agilent Technologies, Inc.) were utilized to analyze the array data for quantile normalization and subsequent data processing. Next, the expression of lncRNAs and mRNAs with at least 6 out of 10 samples flagged as a Present or Marginal 'All Targets Value' were subjected to further data analyses. A statistical difference in the expression of lncRNAs and mRNAs in normal versus tumor samples was identified using volcano plot filtering. Hierarchical clustering analysis was then conducted to obtain the distinguishable expression pattern of IncRNAs and mRNAs between these tissue samples.

Gene Ontology (GO) analysis. GO terms provide the attribution of gene and gene product and interactions in a given organism (http://www.geneontology.org). Once GO terms were obtained, Fisher's exact test was then performed to assess any overlap between the differentially expressed lncRNA 
Table I. Clinicopathological parameters from patients in the screening and validation cohorts.

\begin{tabular}{|c|c|c|c|}
\hline \multirow[b]{2}{*}{ Parameter } & \multirow[b]{2}{*}{ Screening cohort $(\mathrm{n}=5)$} & \multicolumn{2}{|c|}{ Validation cohort } \\
\hline & & $\mathrm{OC}(\mathrm{n}=25)$ & $\mathrm{NT}(\mathrm{n}=15)$ \\
\hline \multicolumn{4}{|l|}{ Age, years } \\
\hline Mean & 52.8 & 55.5 & 56.6 \\
\hline Min-max & $46-60$ & $38-70$ & $43-75$ \\
\hline \multicolumn{4}{|l|}{ Histological type, n (\%) } \\
\hline Serous carcinoma & $2(40)$ & $12(48)$ & n.a \\
\hline Mucinous carcinoma & $2(40)$ & $8(32)$ & n.a \\
\hline Clear-cell carcinoma & $1(20)$ & $3(12)$ & n.a \\
\hline Endometrioid carcinoma & 0 & $2(8)$ & n.a \\
\hline Clinical stage, n (\%) & & & n.a \\
\hline $\mathrm{I} / \mathrm{II}$ & $2(40)$ & $7(28)$ & n.a \\
\hline III/IV & $3(60)$ & $18(72)$ & n.a \\
\hline Grade, n (\%) & & & n.a \\
\hline High & $1(20)$ & $4(16)$ & n.a \\
\hline Mid-low & $4(80)$ & $21(84)$ & n.a \\
\hline Lymph node metastasis, n (\%) & & & n.a \\
\hline No & $4(80)$ & $20(80)$ & n.a \\
\hline Yes & $1(20)$ & $5(20)$ & n.a \\
\hline
\end{tabular}

n.a, not applicable; NT, normal tissue; OC, ovarian cancer.

and the GO annotation list, and $\mathrm{P} \leq 0.05$ was used to show the significance of the GO term enrichment.

Kyoto Encyclopedia of Genes and Genomes (KEGG) gene pathway analysis. Gene pathway enrichment is a functional analysis of the mapped genes using the KEGG tool. This gene pathway analysis was used to better understand the underlying biology of the differentially expressed genes. P-values (Expression Analysis Systematic Explorer-score, Fisher's P-value or hypergeometric P-value) were used to show the significance of a pathway associated with the biological conditions $(\mathrm{P} \leq 0.05)$.

Construction of the coding-non-coding (CNC) gene co-expression network. The link and association between lncRNAs and their coding genes was investigated by constructing the $\mathrm{CNC}$ co-expression network for 4 upregulated and downregulated lncRNAs and their coding genes, respectively. In a given network, the blue nodes represent an mRNA, a red node represents an upregulated lncRNA and a green node represents a downregulated lncRNA. The lines between cycle nodes represent the interactions between an lncRNA and an mRNA, with solid lines indicating a positive correlation and dashed lines indicating a negative correlation. Pearson correlation coefficients between the altered IncRNAs and mRNAs were calculated with a cut-off $\mathrm{P}$-value of $<0.001$ and an absolute value of the correlation coefficient of $\geq 0.99$. The degree values showed the number of genes with which the gene could interact. Those lncRNAs/mRNAs were then further selected to draw the network with Cytoscape (3.1.1) according to a previous study (23).

$R T$ - $q P C R$. Total RNA samples from tissue samples were reverse transcribed into cDNA using the SuperScript ${ }^{\mathrm{TM}}$ III Reverse Transcriptase kit (Invitrogen; Thermo Fisher Scientific, Inc.) according to the manufacturer's protocol. qPCR was then amplified in the ViiA 7 Real-Time PCR system with the Power SYBR Green PCR Master mix (both Applied Biosystems; Thermo Fisher Scientific, Inc.). Briefly, the qPCR mixture was incubated at $95^{\circ} \mathrm{C}$ for $10 \mathrm{~min}$ and then 40 cycles of $95^{\circ} \mathrm{C}$ for $10 \mathrm{sec}$ and $60^{\circ} \mathrm{C}$ for $60 \mathrm{sec}$. The levels of mRNA were normalized to GAPDH and calculated with the $\triangle \mathrm{Cq}$ method. The fold-changes in expression were calculated using the $2^{-\Delta \Delta \mathrm{Cq}}$ method (24). The qPCR primer sequences are shown in Table II. All experiments were performed in triplicate and repeated at least once.

Statistical analysis. Differential levels of lncRNA expression were calculated using fold-change filtration and the independent sample t-test between two groups, while Fisher's exact test was performed for data on the GO and KEGG pathway analyses. $\mathrm{P} \leq 0.05$ was considered to indicate a statistically significant difference. All statistical analyses were conducted using SPSS version 20.0 (IBM Corp., Armonk, NY, USA).

\section{Results}

Profiling of differential expression of lncRNAs in OC. First, differentially expressed lncRNAs in 5 OC and 5 NT samples 
Table II. Reverse transcription-quantitative polymerase chain reaction primer sequences.

\begin{tabular}{llll}
\hline Seq. name & Location & \multicolumn{1}{c}{ Forward primer } & \multicolumn{1}{c}{ Reverse primer } \\
\hline BC041954 & $\mathrm{chr}$ & TCTGTAGTTCGTTGTTGGTCGTG & GCGGTCCTGATTCATTAGCG \\
ENST00000453838 & $\mathrm{chr} 1$ & GCCGGAGTCCTGTCTTCTTATT & TCTGGATGCCTCCCATTTCT \\
ENST00000505048 & $\mathrm{chr} 5$ & ACCCAGGCAGAGGGACAGT & GGCGGCGGTAGGTAGTGAT \\
ENST00000502715 & $\mathrm{chr} 4$ & GACAAGTGGTCTGCCCTGTATG & TGCCTGTTCAACGAGCTATCA \\
AK123324 & $\mathrm{chr}$ & GAGGTTGCGGTGAACTACGAT & CCCATGCTGTGGGATGCT \\
AF087976 & $\mathrm{chr}$ & TTCCAAGTTTCCATTTTCTCACC & AGATTGTTTCTCAGATCCCCAAT \\
AL832916 & $\mathrm{chr} 2$ & CCCCAAACCACTCCAATAGC & CAGCAAGGGCAGAAGGTAAGA \\
AF086261 & $\mathrm{chr15}$ & GCTTTTCTGCTCATGGCTTACA & TGTTGACACCCACTTCCGACT \\
GAPDH & & GGGAAACTGTGGCGTGAT & GAGTGGGTGTCGCTGTTGA \\
\hline
\end{tabular}

Seq., sequence.

were profiled using the Arraystar Human LncRNA Microarray. These differentially expressed lncRNAs were calculated using the log fold-change between tumor and NT samples, with a cut-off point of 1:2.5. A positive value indicated the upregulated genes in the tumor, whereas a negative value indicated downregulated genes in tumor versus normal samples.

The expression of 25,256 lncRNAs was detected in the 5 paired OC and NT samples, and the box plot view was used to compare their distributions in the OC and NT samples, as shown in Fig. 1A, while differentially expressed lncRNAs in OC samples relative to NT controls are shown in Fig. 1B. The hierarchical clustering analysis showed a distinguishable lncRNA expression profile between the OC and NT samples (Fig. 1C).

Moreover, volcano plots were constructed using fold-change data, which enabled the visualization of the association between the fold-change and statistical significance. A total of 2,870 differentially expressed lncRNAs were identified between OC and NT (Fig. 1D). Of these 2,870 dysregulated lncRNAs, 795 were upregulated and 2,075 were downregulated in OC. The top 10 most upregulated lncRNAs in OC were BC041954, ENST00000423200,uc.428+, BC028018,ENST00000433201, ENST00000458624, ENST00000453838, CR601061, ENST00000505048 and ENST00000502715, whereas the top 10 most downregulated lncRNAs in OC were AK123324, AF087976, NR_001284, ENST00000474313, AL832916, AF086261, BC070168, uc001zfv.1, NR_023313 and uc002btm.2 (Table III). BC041954 (fold-change of 177.98) was the most significantly upregulated gene, and AK123324 (fold-change of 56.42) was the most significantly downregulated lncRNA. Overall, upregulated lncRNAs were less common in OC than downregulated lncRNAs in the microarray data. Next, 4 upregulated and 4 downregulated lncRNAs were selected for validation (BC041954, ENST00000453838, ENST00000505048, ENST00000502715, AK123324, AF087976, AL832916, and AF086261).

Profiling of differential expression of $m R N A$ s in $O C$. The array analysis identified 19,362 mRNAs of 30,215 coding transcripts probes (Fig. 2A and B) between OC and NT, among which 2,658 mRNAs were aberrantly expressed in OC (Fig. 2C). Of the 2,658 differentially expressed mRNAs in the OC
Table III. List of 10 differentially expressed lncRNAs in ovarian cancer analyzed by the microarray.

A, Upregulated lncRNAs

\begin{tabular}{lcc}
\hline lncRNA seq name & Fold-change & P-value \\
\hline BC041954 & 177.97792 & $2.160 \times 10^{-3}$ \\
ENST00000423200 & 68.051605 & $4.842 \times 10^{-6}$ \\
uc.428+ & 50.279484 & $1.748 \times 10^{-5}$ \\
BC028018 & 34.942886 & $7.128 \times 10^{-6}$ \\
ENST00000433201 & 26.048214 & $2.346 \times 10^{-3}$ \\
ENST00000458624 & 20.718157 & $5.804 \times 10^{-7}$ \\
ENST00000453838 & 19.044817 & $8.763 \times 10^{-6}$ \\
CR601061 & 18.448626 & $1.821 \times 10^{-5}$ \\
ENST00000505048 & 17.240042 & $3.885 \times 10^{-5}$ \\
ENST00000502715 & 17.071527 & $2.145 \times 10^{-3}$
\end{tabular}

B, Downregulated lncRNAs

\begin{tabular}{lll}
\hline AK123324 & 56.423824 & $1.524 \times 10^{-3}$ \\
AF087976 & 40.975834 & $1.436 \times 10^{-5}$ \\
NR_001284 & 36.627293 & $1.017 \times 10^{-3}$ \\
ENST00000474313 & 35.2125 & $5.514 \times 10^{-4}$ \\
AL832916 & 31.709267 & $7.592 \times 10^{-3}$ \\
AF086261 & 29.65302 & $9.410 \times 10^{-6}$ \\
BC070168 & 28.347292 & $8.506 \times 10^{-3}$ \\
uc001zfv.1 & 25.797493 & $9.822 \times 10^{-4}$ \\
NR_023313 & 24.778913 & $4.815 \times 10^{-3}$ \\
uc002btm.2 & 24.472792 & $7.887 \times 10^{-4}$ \\
\hline
\end{tabular}

lncRNA, long non-coding RNA; seq, sequence.

samples, 1,014 mRNA were found to be upregulated and 1,644 mRNA to be downregulated compared with those of the NT (Table IV). The hierarchical clustering analysis showed a significant association of their expression patterns between the OC and NT samples (Fig. 2D). 

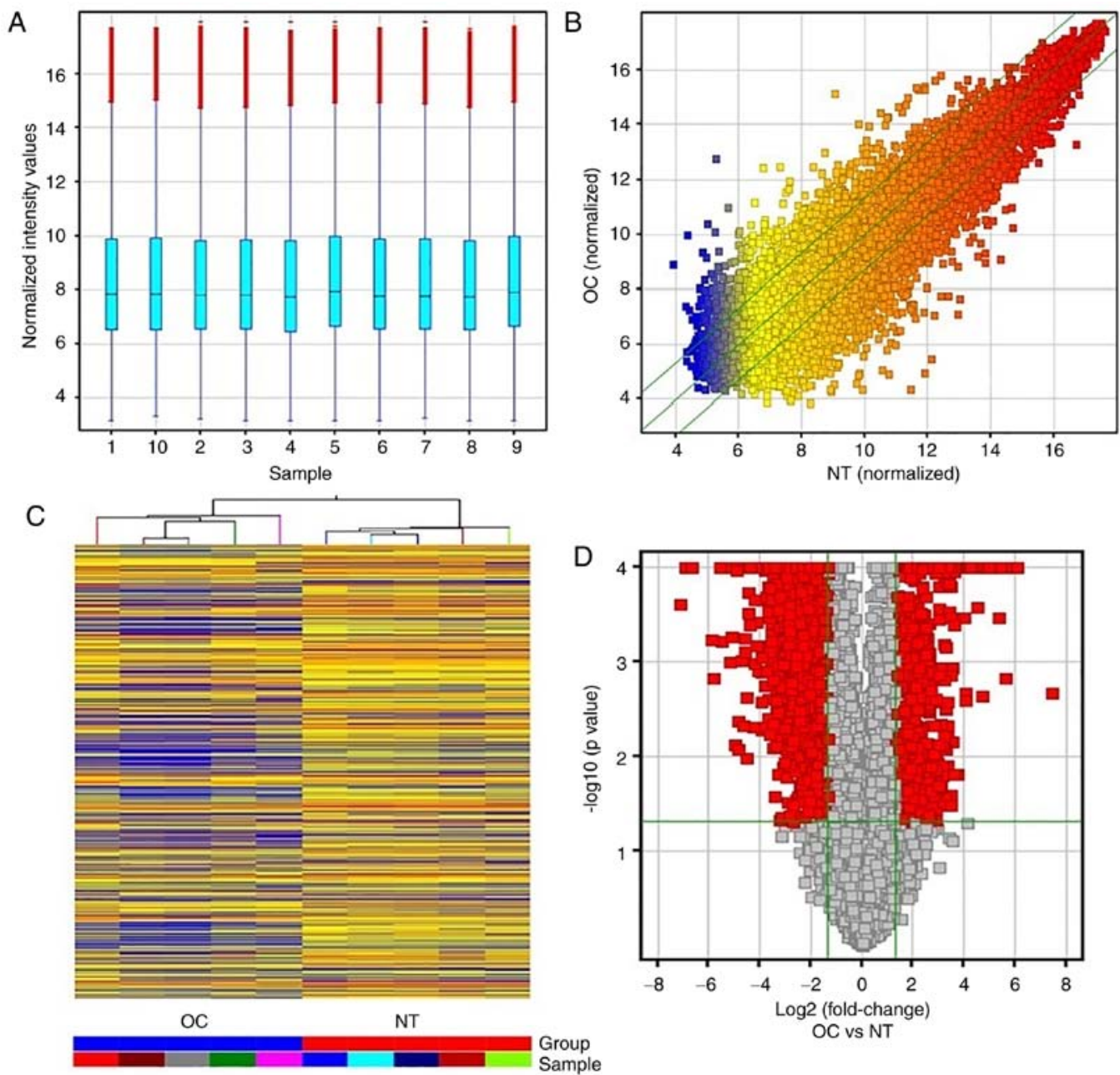

Figure 1. Microarray profile of differential lncRNA expression in OC compared with NT samples. (A) Box plot: Comparison of the distribution of lncRNA expression values in OC versus NT samples following normalization to the system control. (B) Scatter plot of variations in expression of lncRNAs between OC and NT samples. (C) Hierarchical clustering analysis of differentially expressed lncRNAs between OC and NT samples. (D) The volcano plot of differently expressed lncRNAs in OC versus NT samples. The vertical lines correspond to 2.5 -fold changes in the upregulation and downregulation of lncRNAs, respectively, whereas the horizontal lines represent a P-value of $<0.05$. The red dots represent the differentially expressed lncRNAs with statistical significance. OC, ovarian cancer; NT, normal tissue; lncRNA, long non-coding RNA.

Identification of genes and gene pathways using GO and KEGG pathway analyses. To systematically investigate the potential functions of these target genes of differentially expressed lncRNAs in OC, GO and KEGG pathway analyses were performed. The data showed that these upregulated genes were involved in the development of the "cell anatomical structure' (GO:0048856; $\left.\mathrm{P}=5.46187 \times 10^{-6}\right)$, 'developmental process' (GO:0032502; $\mathrm{P}=1.04997 \times 10^{-5}$ ), 'system development' (GO:0048731; $\mathrm{P}=1.47343 \times 10^{-5}$ ) and 'multicellular organismal development' (GO:0007275; P=4.63519x10 ${ }^{-5}$ ) (Fig. 3A). By contrast, these downregulated genes were involved in 'gene expression' (GO: 0010476; $\mathrm{P}=1.8095 \times 10^{-6}$ ), 'nitrogen compound metabolic process' (GO:0006807; $\mathrm{P}=2.80064 \times 10^{-6}$ ), 'kidney development' (GO:0001822; $\left.\mathrm{P}=4.23279 \times 10^{-6}\right)$ and 'cellular nitrogen compound metabolic processes' (GO:0034641; $\mathrm{P}=6.77475 \times 10^{-6}$ ) (Fig. 3B). In addition, it was also found that the highest enriched GO terms in the upregulated transcripts were the 'plasma membrane compartment' (GO:0044459; $\mathrm{P}=3.68 \times 10^{-5}$ ) for cellular components (Fig. 3C) and 'scavenger receptor activity' (GO:0005044; $\mathrm{P}=2.17 \times 10^{-4}$ ) for molecular functions (Fig. 3D). The highest enriched GO terms in the downregulated transcripts were the 'intracellular compartment' (GO:0044424; $\mathrm{P}=7.26 \times 10^{-10}$ ) for cellular components (Fig. 3E) and 'glutathione transferase activity (GO:0004364; $\mathrm{P}=1.22 \times 10^{-6}$ ) for molecular functions (Fig. $3 \mathrm{~F}$ ).

Furthermore, the KEGG gene pathway analysis revealed that these targeting genes participated in several signaling pathways; for example, these upregulated transcripts could form 12 gene pathways and were involved and enriched in 'Staphylococcus aureus infection', 'asthma', 'systemic lupus erythematosus' and 'sphingolipid metabolism' (Fig. 4A). By contrast, the downregulated transcripts could form 33 pathways that corresponded to and were primarily enriched in the 'metabolism of xenobiotics by cytochrome $\mathrm{P} 450$ '. The other main pathways included 'drug metabolism-cytochrome P450', 'fatty acid metabolism' and 'propanoate metabolism' (Fig. 4B).

Identification of the CNC gene co-expression network. To further assess the lncRNAs to directly regulate the expression of target mRNAs, a CNC gene co-expression network was generated based on the differentially expressed lncRNA and targeted genes. Using these 4 upregulated and downregulated 

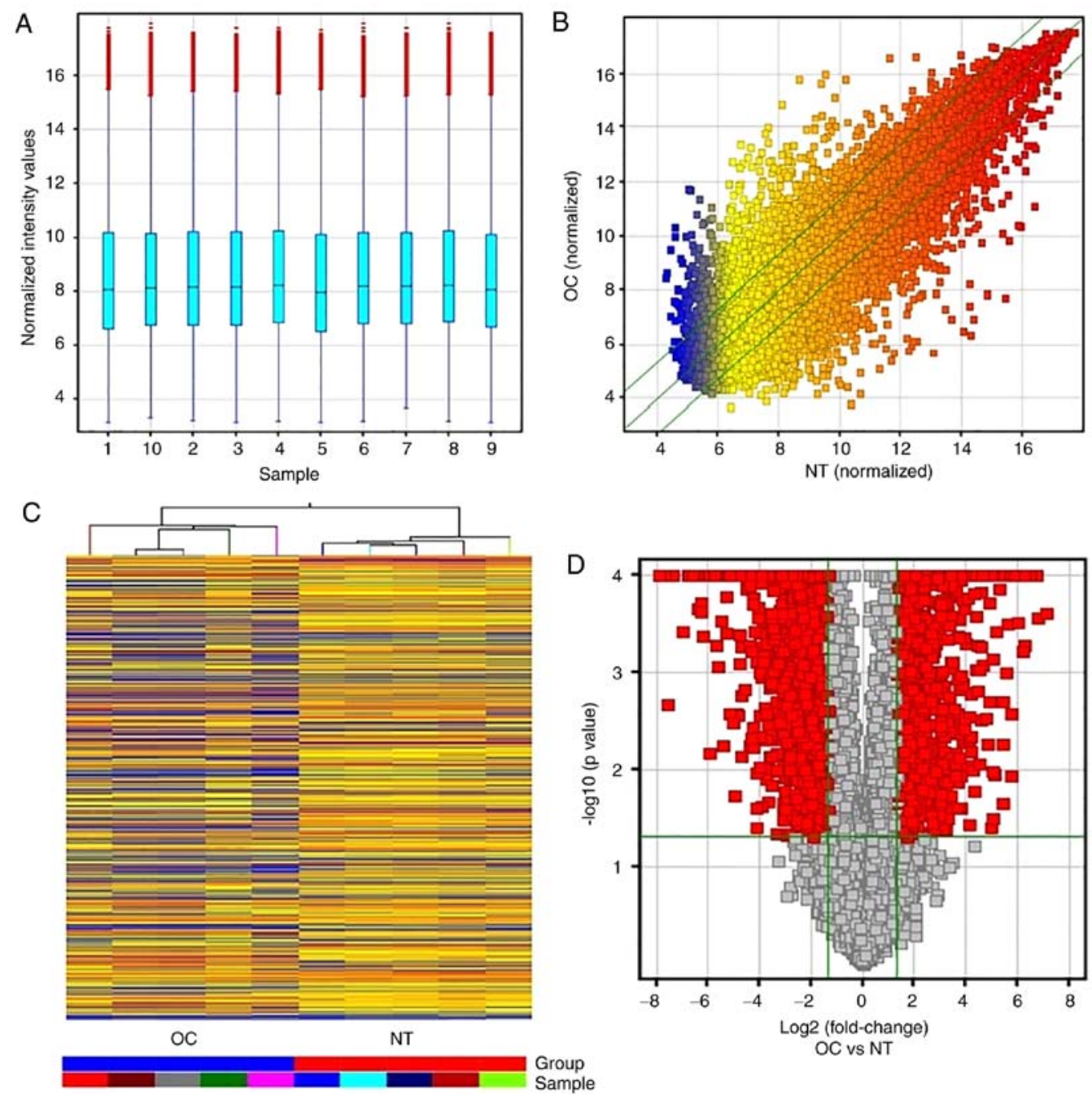

Figure 2. Microarray profile of differential mRNA expression in OC compared with NT samples. (A) Box plot: Comparison of the distributions of mRNA expression values in OC versus NT samples following normalization to the system control. (B) Scatter plot of variation in mRNA expression between OC and NT samples. (C) Hierarchical clustering analysis of differentially expressed mRNAs between OC and NT tissues. (D) Volcano plot of differently expressed mRNAs in OC relative to NT samples. The vertical lines correspond to 2.5 -fold changes in upregulation and downregulation of mRNAs, respectively, whereas the horizontal lines represent a P-value of $<0.05$. The red dots represent the differentially expressed mRNAs with statistical significance. OC, ovarian cancer; NT, normal tissue; lncRNA, long non-coding RNA.

lncRNAs, a CNC network was constructed and it was found that among the upregulated co-expression network, 4 lncRNAs and 307 mRNAs were comprised the CNC network nodes, resulting in 202 positive pairs and 202 negative pairs (Fig. 5). However, among the downregulated co-expression network, 4 lncRNAs and 460 mRNAs comprised the CNC network node, leading to 454 positive pairs and 184 negative pairs (Fig. 6). As shown in the figures, the blue nodes represent mRNA (protein-coding gene), the red nodes represent the upregulated lncRNA and the green nodes represent the downregulated lncRNA (the non-coding gene). The lines between the cycle nodes represent the interactions between lncRNA and mRNA. Solid lines indicate a positive correlation, whereas dashed lines indicate a negative correlation. The CNC network indicates the interregulation of lncRNAs and mRNAs for OC development.

Classification and subgroups of differentially expressed lncRNAs. These differentially expressed lncRNAs were further classified and subgrouped into four classes of lncRNA, namely, the enhancer IncRNA nearby coding gene, HOX cluster, long-intergenic non-coding RNAs (lincRNAs) nearby coding gene and Rinn lincRNAs. The enhancer IncRNA nearby coding gene contained the differentially expressed enhancer-like lncRNAs and their nearby coding genes with DNA sequence distances of $<300 \mathrm{~kb}$, while the HOX cluster lncRNAs contained four HOX loci targeting 407 discrete transcribed regions according to a previous study by Rinn et al (25). Moreover, lincRNAs nearby coding genes contained the differentially expressed lincRNAs, as did nearby coding gene pairs with a DNA sequence distance of $<300 \mathrm{~kb}$. The Rinn lincRNAs profile data contained data for lncRNAs based on a study by Rinn et al (25). Overall, the current study identified 36 enhancer lncRNAs nearby coding genes, 507 HOX clusters, 90 lincRNAs nearby coding genes and 2,606 Rinn lincRNAs in OC compared with those of NT.

Validation of microarray data. Although microarray data provide a global assessment of transcriptomic variations, their resolution and accuracy may be limited at the 


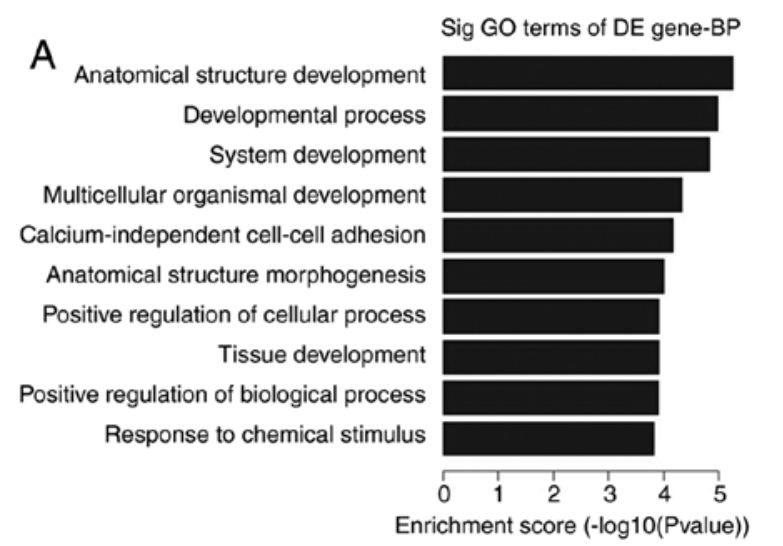

C

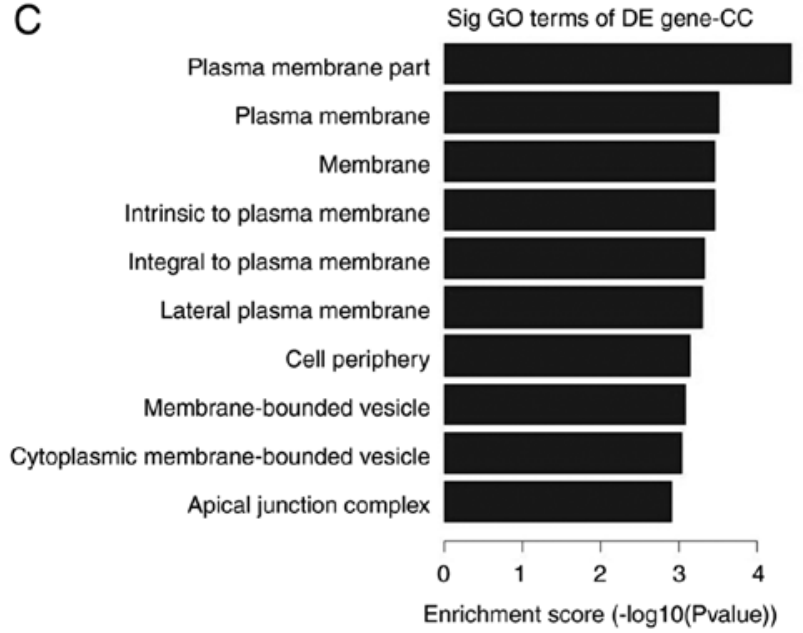

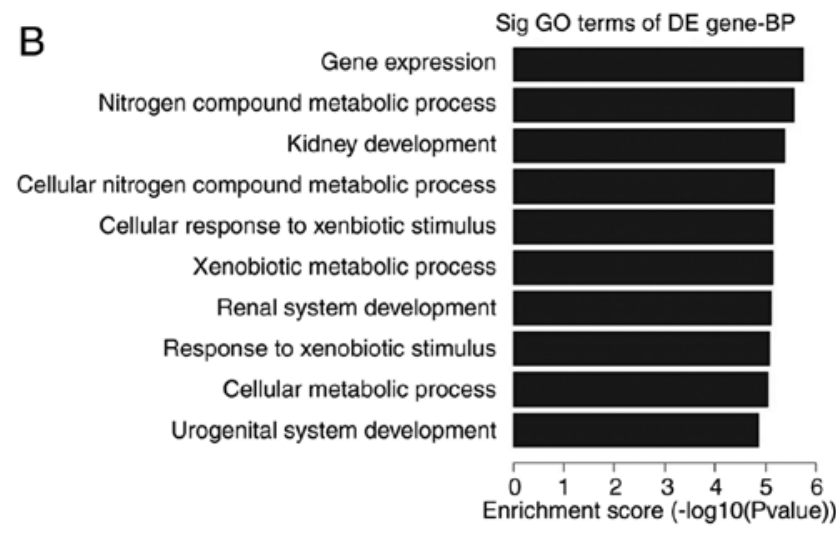

D
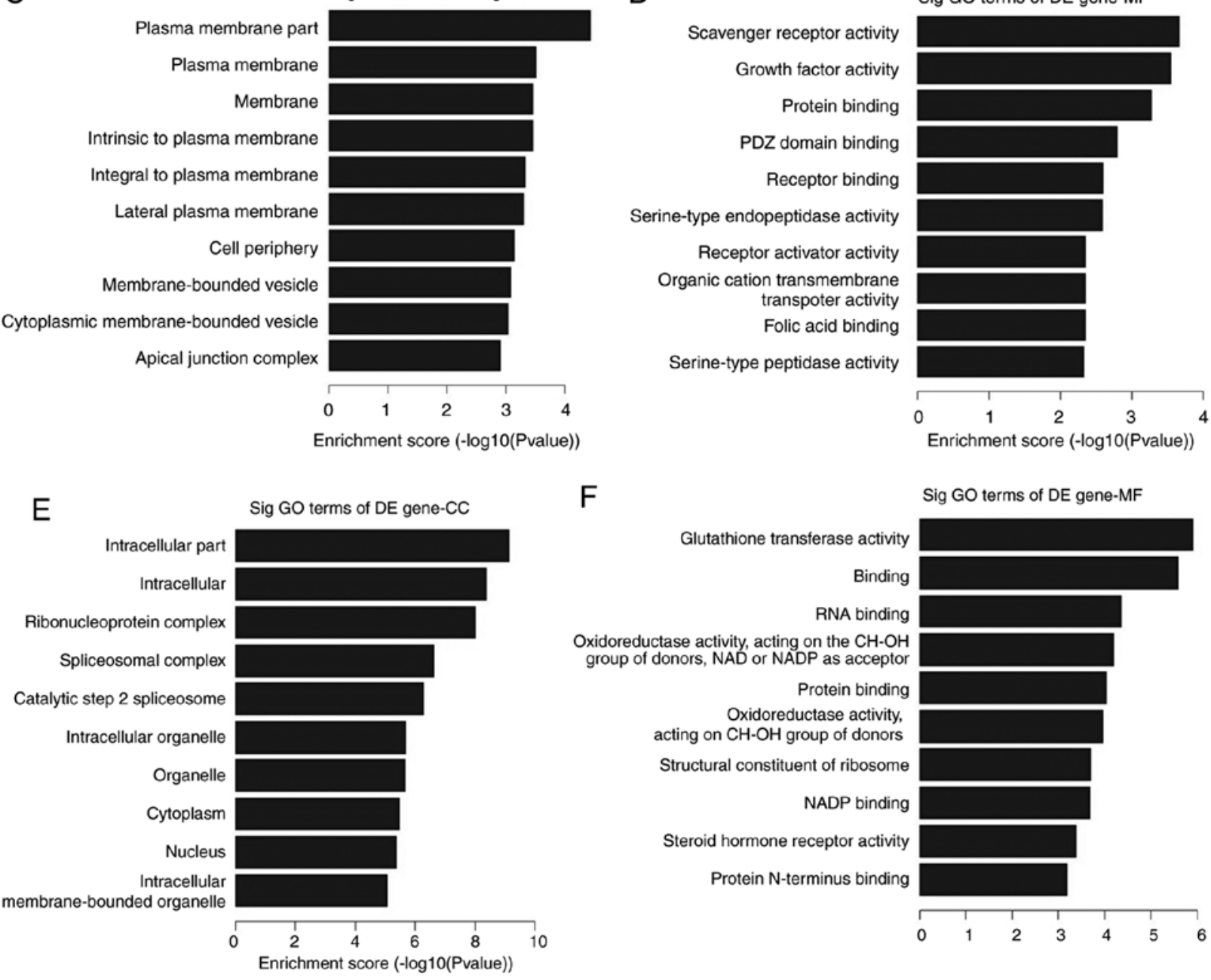

$\mathrm{F}$

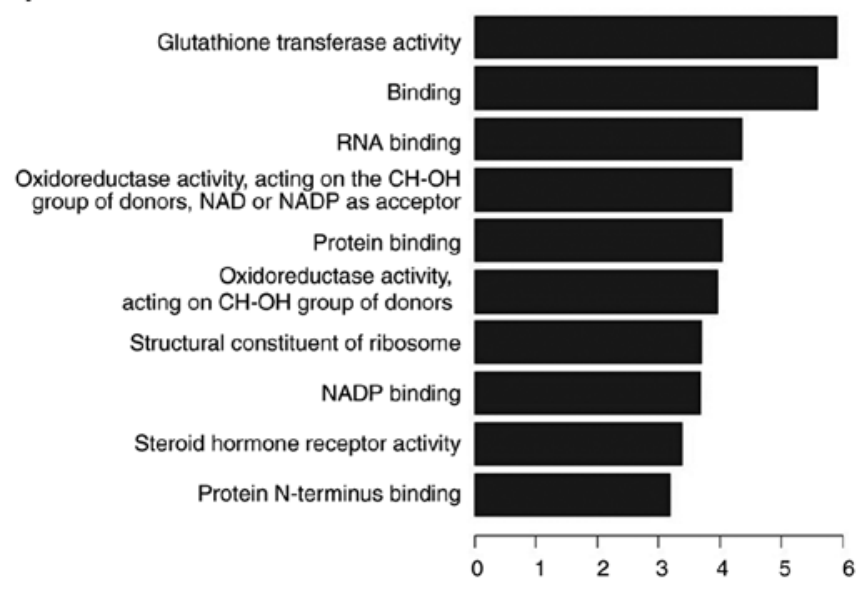

Figure 3. GO analysis of lncRNAs targeting genes. (A) The top $10 \mathrm{GO}$ terms of biological processes in the upregulated lncRNAs in OC. (B) The top $10 \mathrm{GO}$ terms of biological processes in the downregulated lncRNAs in OC. (C) The top $10 \mathrm{GO}$ terms of cellular components in upregulated lncRNAs in OC. (D) The top $10 \mathrm{GO}$ terms of molecular functions in upregulated lncRNAs in OC. (E) The top $10 \mathrm{GO}$ terms of cellular components in downregulated lncRNAs in OC. (F) The top $10 \mathrm{GO}$ terms of molecular functions in downregulated lncRNAs in OC. OC, ovarian cancer; GO, Gene Ontology; lncRNA, long non-coding RNA; $\mathrm{CC}$, cellular components; MF, molecular functions; BP, biological processes; Sig, significant; DE, differentially expressed.

individual gene level (26). RT-qPCR analysis was thus performed to validate certain microarray data using this set of tissue samples. Overall, 4 of the most markedly upregulated (BC041954, ENST00000453838, ENST00000505048 and ENST00000502715) and downregulated (AK123324, AF087976, AL832916 and AF086261) lncRNAs were selected. The results showed that, with the exception of
ENST00000453838 and ENST00000505048, all other IncRNAs were consistent with those of the microarray data (Figs. 7 and 8).

One of these differently expressed lncRNAs (BC041954) was further assessed using independent tissue samples containing $25 \mathrm{OC}$ and $15 \mathrm{NT}$ (Fig. 9). The RT-qPCR results further supported the microarray data. 
A

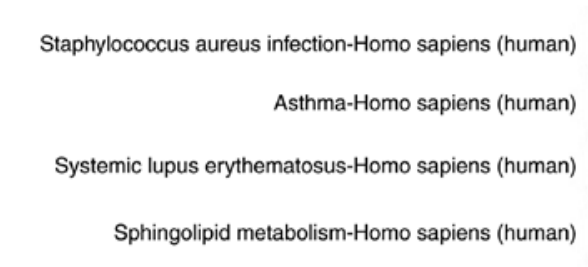

Leukocyte transendothelial migration -Homo sapiens (human)

Bladder cancer-Homo sapiens (human)

Cell adhesion molecules (CAMs)-Homo sapiens (human)

Regulation of actin cytoskeleton-Homo sapiens (human)

Phagosome-Homo sapiens (human)

Cell cycle-Homo sapiens (human)

\section{B}

Metabolism of xenobiotics by cytochrome P450-Homo sapiens (human)

Drug metabolism-cytochrome P450-Homo sapiens (human)

Fatty acid metabolism-Homo sapiens (human)

Propanoate metabolism-Homo sapiens (human)

MAPK signaling pathway-Homo sapiens (human)

Glutathione metabolism-Homo sapiens (human)

Neurotrophin signaling pathway-Homo sapiens (human)

Ribosome-Homo sapiens (human)

Valine, leucine and isoleucine degradation-Homo sapiens (human)

Butanoate metabolism-Homo sapiens (human)
Sig pathway of DE gene

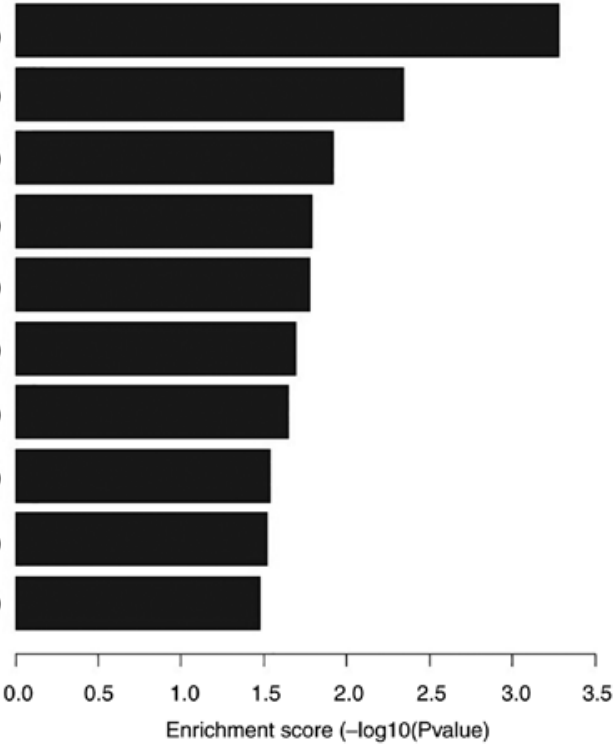

Sig pathway of DE gene

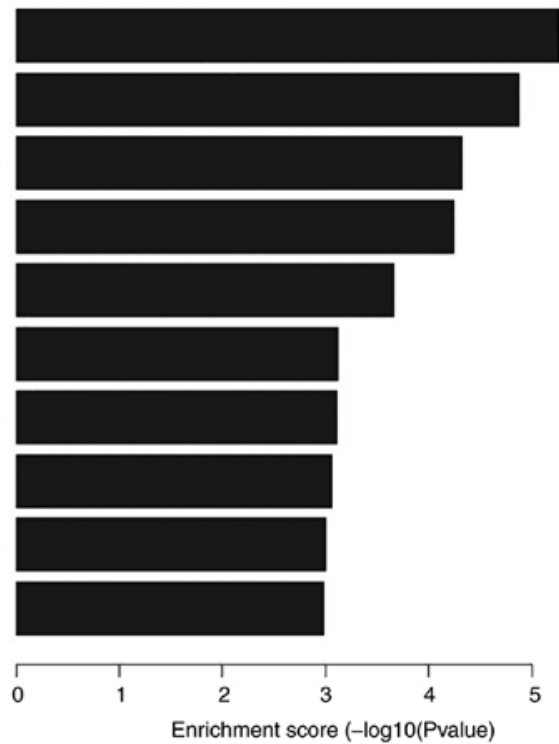

Figure 4. Kyoto Encyclopedia of Genes and Genomes pathway analysis of lncRNAs targeting gene pathways. The data show the top 10 pathways with significant differences between ovarian cancer and normal tissues. (A) Upregulated lncRNA-related gene pathways. (B) Downregulated lncRNA-related gene pathways. Sig, significant; DE, differentially expressed; lncRNA, long non-coding RNA.

\section{Discussion}

The current study profiled differentially expressed lncRNAs and mRNAs in OC versus NT and found 2,870 dysregulated lncRNAs (795 were upregulated and 2,075 were downregulated in OC). There were 2,658 differentially expressed mRNAs in OC (1,014 were upregulated and 1,644 were downregulated compared with those of NT). The RT-qPCR analysis further confirmed the majority of the microarray data. Furthermore, the GO analysis showed that the upregulated genes were involved in the development of the 'cell anatomical structure', 'embryo and system development', and 'multicellular organismal development'. By contrast, the downregulated genes were involved in 'gene expression', 'nitrogen compound metabolic processes', 'kidney development' and 'cellular nitrogen compound metabolic processes'. The KEGG pathway analysis revealed that the upregulated transcripts could form 12 gene pathways, but that the downregulated transcripts could form 33 pathways. These differentially expressed lncRNAs could be classified into four classes of IncRNA, namely, the enhancer lncRNA nearby coding gene, HOX cluster, lincRNAs nearby a coding gene and Rinn lincRNAs. The CNC gene co-expression network data showed the interregulation of IncRNAs and mRNAs for OC development. In conclusion, the expression of lncRNAs and mRNAs was dysregulated in $\mathrm{OC}$, and further investigation could provide novel information for these dysregulated genes as biomarkers, therapeutic targets, and/or molecular mechanisms of OC.

Notably, lncRNAs are a class of regulatory molecules for which aberrant expression was associated with malignant transformation of various normal cells (27). lncRNAs regulate 

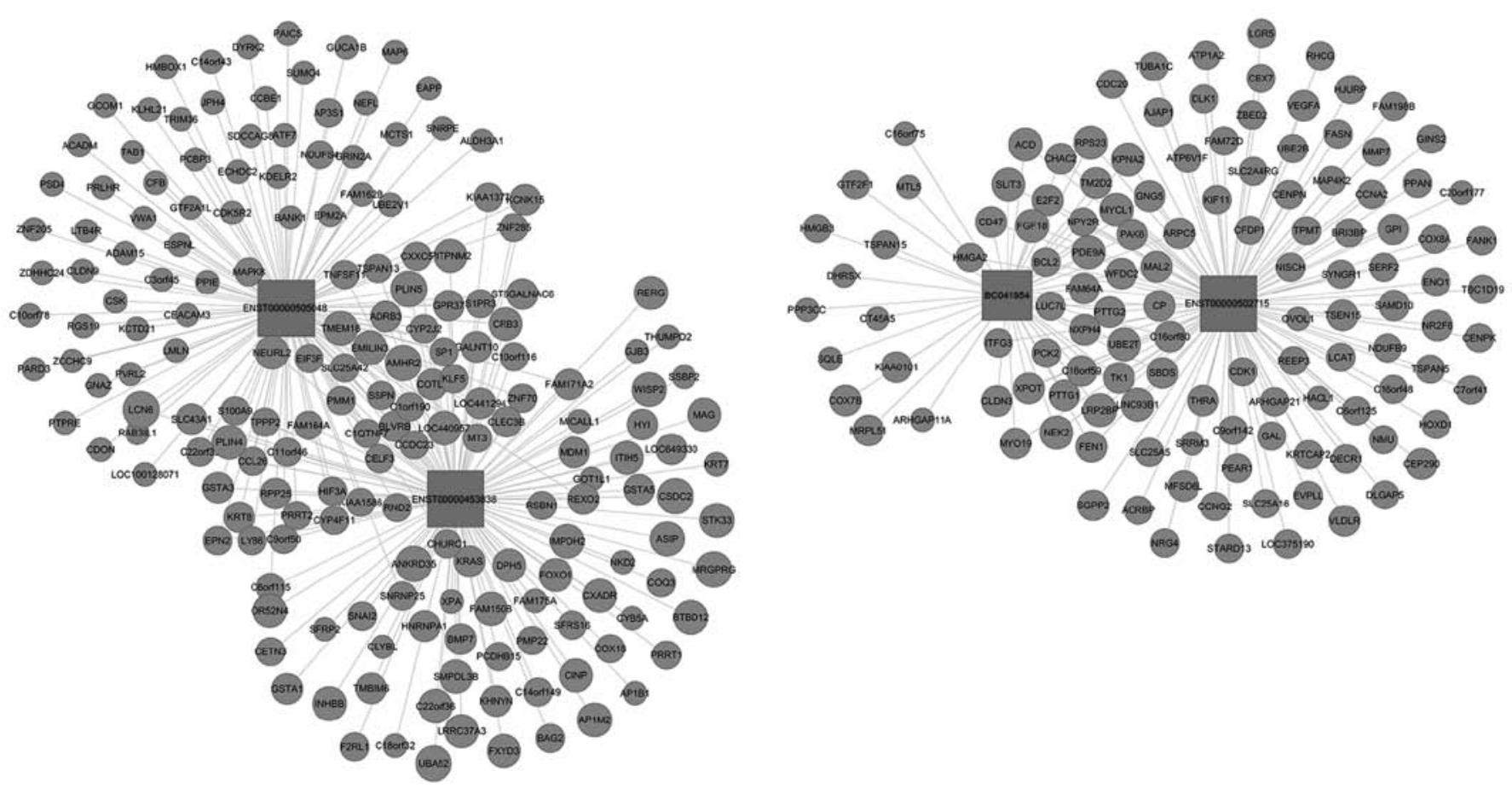

Figure 5. Coding-non-coding gene network analysis of the interaction between lncRNAs, and the targeting gene network using the 4 upregulated lncRNAs. lncRNA, long non-coding RNA.

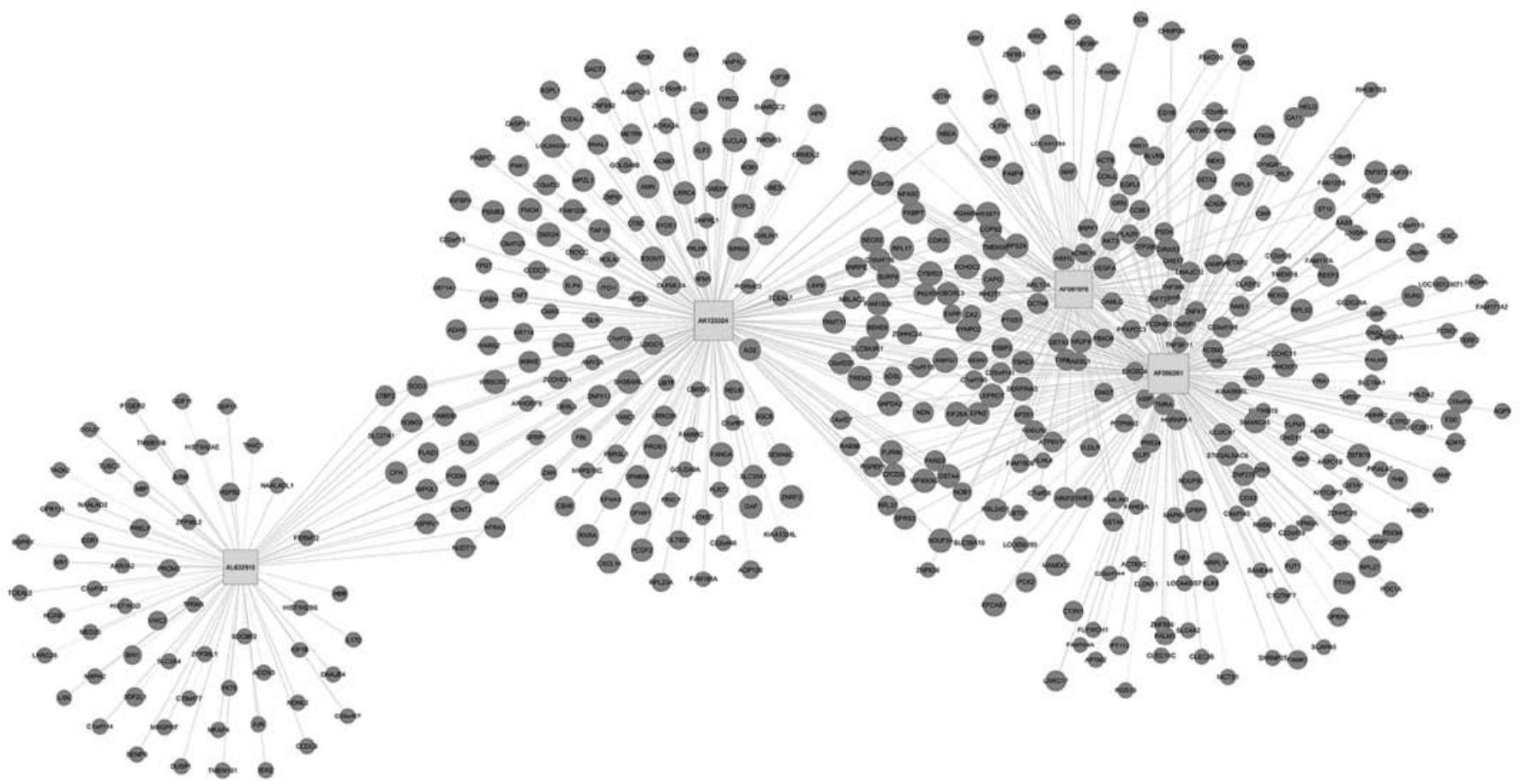

Figure 6. Coding-non-coding gene network analysis of the interaction between lncRNAs, and the targeting gene network using the 4 downregulated lncRNAs. lncRNA, long non-coding RNA.

the expression of their targeting genes and are associated with cancer development (17-20). Certain IncRNAs function as tumor oncogenes, whereas others function as tumor suppressors $(17,19,20)$. In OC, previous studies have shown that lncRNAs serve a crucial role in malignant OC $(10,13,14,16,17)$. For example, recent studies showed that hepatocellular carcinoma upregulated lncRNA could function as an oncogene to target the expression of autophagy related 7 and integrin subunit $\beta 1$ (28), and lncRNA Ewing sarcoma-associated transcript 1 was able to promote OC progression by targeting miR-330-5p expression (29). Meanwhile, the lost expression of IncRNA SPRY4 intronic transcript 1 promoted OC cell metastasis partly by inducting tumor cell epithelial-mesenchymal transition (30) and reducing the expression of 
Table IV. List of 10 differentially expressed mRNAs in ovarian cancer analyzed by the microarray.

A, Upregulated mRNAs

\begin{tabular}{lcc}
\hline Gene symbol & Fold-change & P-value \\
\hline CLDN3 & 142.23268 & $2.499 \times 10^{-4}$ \\
FOLR1 & 112.25475 & $2.825 \times 10^{-4}$ \\
OVOL2 & 104.51445 & $7.898 \times 10^{-6}$ \\
PVRL4 & 97.82674 & $3.914 \times 10^{-5}$ \\
SMPDL3B & 96.442276 & $6.986 \times 10^{-6}$ \\
CLDN4 & 89.58858 & $8.086 \times 10^{-6}$ \\
BMP7 & 81.024956 & $2.833 \times 10^{-5}$ \\
AQP5 & 77.35999 & $5.248 \times 10^{-4}$ \\
DOK7 & 76.89189 & $5.335 \times 10^{-5}$ \\
SCGB2A1 & 73.11675 & $1.762 \times 10^{-5}$ \\
\hline
\end{tabular}

B, Downregulated mRNAs

\begin{tabular}{lcc}
\hline STAR & 242.25418 & $3.644 \times 10^{-5}$ \\
WFIKKN2 & 208.04768 & $1.603 \times 10^{-5}$ \\
VWC2 & 188.6573 & $2.201 \times 10^{-3}$ \\
PROK1 & 172.80093 & $1.872 \times 10^{-6}$ \\
ASIP & 167.52657 & $5.426 \times 10^{-8}$ \\
FOXL2 & 124.60296 & $3.792 \times 10^{-4}$ \\
CCBE1 & 113.107506 & $1.352 \times 10^{-5}$ \\
FAM150B & 104.271996 & $1.048 \times 10^{-7}$ \\
PCDH11Y & 97.41786 & $1.174 \times 10^{-5}$ \\
GATM & 90.50611 & $3.095 \times 10^{-5}$ \\
\hline
\end{tabular}

IncRNA tubulin $\alpha 4 \mathrm{~b}$, which was associated with poor EOC prognosis (31). Another recent study showed that an integrated miRNA-lncRNA signature was able to predict survival in patients with wild-type BRCA1 DNA repair associated/BRCA2 DNA repair associated OC (32). Moreover, the altered lncRNA signature may also predict paclitaxel resistance in EOC patients (33). In addition, the lncRNA colon cancer-associated transcript 1 was shown to associate with poor prognosis in EOC patients by promoting tumor metastasis (34). The current study found a great number of differentially expressed lncRNAs in OC tissues versus NT. Notably, previous microarray profiling studies also showed altered lncRNA expression in OC $(35,36)$. Thus, detecting aberrant lncRNA expression could potentially be a novel technique for cancer diagnosis and prognosis.

However, the microarray profile analysis is only performed to globally assess altered gene expression in samples, and a validation set of samples is required to verify the microarray data. The current study confirmed the majority of the microarray data, as well as the most upregulated and downregulated lncRNAs in OC tissues, further suggesting that the differently expressed lncRNAs may be involved in the molecular regulation of OC development. BC041954 was selected from among the markedly upregulated IncRNAs for further validation using RT-qPCR in 25 OC and 15 NT samples, and the data confirmed alterations of lncRNA expression in OC. Furthermore, GO,

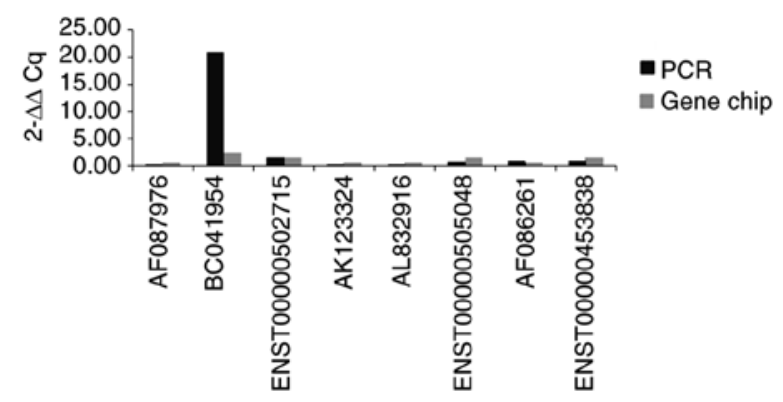

Figure 7. Comparison of lncRNA expression level between microarray and RT-qPCR data. A total of 4 upregulated and 4 downregulated lncRNAs were validated in 5 ovarian cancer and 5 normal tissue samples using RT-qPCR. With the exception of ENST00000453838 and ENST00000505048, the data for the lncRNAs were consistent with the microarray data. IncRNA, long non-coding RNA; RT-qPCR, reverse transcription-quantitative polymerase chain reaction.

KEGG pathway and co-expression network analyses were utilized to investigate the potential functions of these target genes of differentially expressed lncRNAs in OC. It was found that based on the GO analysis, the top GO term of the upregulated lncRNAs was 'anatomical structure development', whereas the top GO term of the downregulated IncRNAs was 'gene expression'. These results indicated that lncRNAs regulate gene expression for their biological function. In addition, the KEGG pathway analysis identified 45 pathways that corresponded to differentially expressed transcripts. Among them, 12 pathways were in upregulated transcripts and 33 pathways in downregulated transcripts. The upregulated lncRNAs were correlated with cell adhesion molecules at the cell surface to regulate the cell-extracellular matrix and specific cell-cell interactions (37). Various studies have reported that adhesion molecules serve critical roles in tumor development and metastasis (38). For instance, the IncRNA BC048612 can regulate the expression of neuronal growth regulator 1 (NEGR1), a cell adhesion molecule, and a recent study (37) demonstrated that the IncRNA BC048612 transcribed from the bi-directional GC-rich promoter of NEGR1 was able to upregulate NEGR1 expression, whereas the knockdown of BC048612 expression led to the significant downregulation of NEGR1 expression. Another study (38) showed that the IncRNA LINC00152 could activate the rapamycin (mechanistic target of rapamycin) pathway by binding to the epithelial cell adhesion molecule promoter. Therefore, we speculated that there may be a link between the dysregulated IncRNAs and adhesion molecules in OC tumorigenesis.

Furthermore, according to the link between lncRNAs and their targeting protein-coding genes, IncRNAs can be characterized as antisense, intergenic, enhancer, bidirectional and intronic lncRNAs. The present study next conducted a subgroup analysis and revealed four classes of IncRNA, namely, the enhancer IncRNA nearby coding gene, HOX cluster, lincRNAs nearby coding gene and Rinn lincRNAs. Various lncRNAs are able to exert their biological function as enhancers, i.e., the activation of gene transcription by serving as cis-regulatory molecules. The enhancer lncRNA is transcribed from the sequences of the enhancer regions to actively regulate the transcription of the protein-coding genes (16). A recent study demonstrated that a number of enhancer 


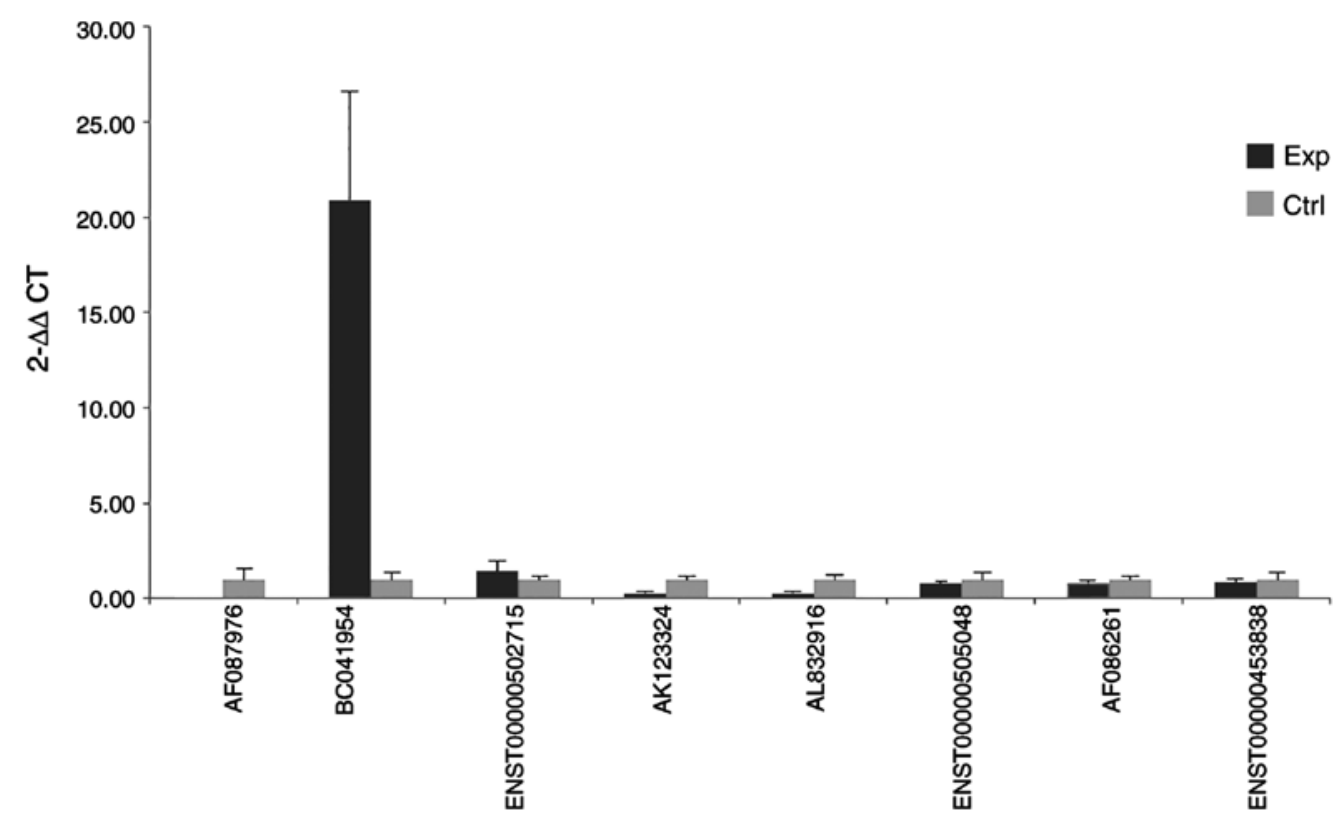

Figure 8. RT-qPCR validation of lncRNAs in ovarian cancer and normal tissue samples. Levels $(\mathrm{P}<0.05)$ of 8 differentially expressed lncRNAs were verified using RT-qPCR in 5 ovarian cancer and 5 normal tissue samples. IncRNA, long non-coding RNA; RT-qPCR, reverse transcription-quantitative polymerase chain reaction.

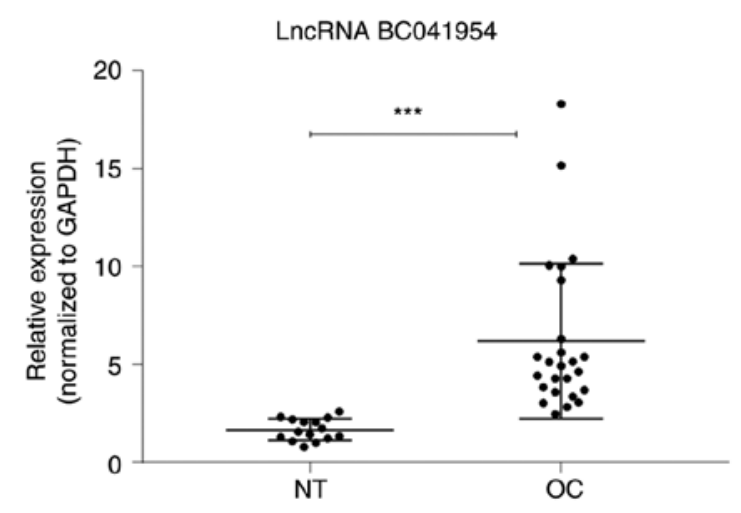

Figure 9. RT-qPCR validation of one candidate lncRNA in OC and NT. The expression of lncRNA BC041954 was assessed using RT-qPCR in 25 OC and 15 NT samples. The dots represent the fold-changes in lncRNA expression intensity in OC versus NT, whereas the horizontal lines show the maximal, minimal and median values, respectively, of the fold-changes in each sample set $\left({ }^{* * *} \mathrm{P}<0.001\right)$. lncRNA, long non-coding RNA; RT-qPCR, reverse transcription-quantitative polymerase chain reaction; $\mathrm{OC}$, ovarian cancer; NT, normal tissue.

elements were transcribed and produced RNA molecules, as the enhancer RNAs (39). By contrast, lincRNAs include a great number of the non-coding regions interspersed between genes $(10,16,27)$ and function in various cell processes, including embryonic stem cell pluripotency, cell growth, or tumor metastasis $(7,23)$. The current study revealed that BC041954 was the most significantly upregulated lncRNA in OC compared with NT. BC041954 is 1,055 bp in length and localized at chromosome 3 near the Zic family member 4 gene. lncRNA ENST00000423200 is a 342-bp intergenic lncRNA localized in chromosome 4. IncRNA ENST00000433201 is a 531-bp intergenic lncRNA localized at chromosome 15, close to the AC104759.2 gene. By contrast, the current study showed that lncRNA AK123324, which has 2,568 bp as an intergenic lncRNA at chromosome 8, was the most significantly downregulated in OC, while AF087976 is a 652-bp intergenic lncRNA localized at chromosome 6. The current study revealed several novel lncRNAs; however, thus far, they not been found to be altered in OC or other cancer types.

There are certain limitations to the current study; for example, the profiles of the differentially expressed lncRNAs varied among the different microarray profiling studies, and the sample size was relatively small in the current study, which could lead to such a difference. The expression of various lncRNAs could be highly tissue-specific and cancer-specific (37). Therefore, the signature of lncRNAs as a biomarker for tumor diagnosis and prognosis requires further confirmation with independent larger sample sizes of patients in future studies. Moreover, more functional analysis of the role of IncRNAs in OC is required.

In summary, the current study profiled the differential lncRNA and mRNA expression between OC and NT samples, and revealed that these IncRNAs and $m R N A s$ may serve a role in OC. The comprehensive GO and KEGG pathway and CNC network analyses indicated that certain lncRNAs/mRNAs may serve important roles in OC pathogenesis. These data may offer novel insights into $\mathrm{OC}$ pathogenesis and could be a promising strategy to dissect the molecular mechanism of OC. Further studies are warranted to provide convincing evidence for clarifying the functions of lncRNA in OC.

\section{Acknowledgements}

Not applicable.

\section{Funding}

This study was supported in part by grants from National Natural Science Foundation of China (no. 81001163), 
The Natural Science Foundation of Liaoning Province (no. 2015020462) and Shenyang Science and Technology Project Funds (no. F13-220-9-46).

\section{Availability of data and materials}

The datasets used and/or analyzed during the current study are available from the corresponding author on reasonable request.

\section{Authors' contributions}

YML: System design, paper writing and modification of the experimental design. YW: Microarray analysis. SQL: Collection of ovarian specimens, RT-qPCR and related data analysis. MYZ: GO analysis, pathway analysis and related data analysis. YRG: CNC analysis and related data analysis. All authors read and approved the final manuscript.

\section{Ethics approval and consent to participate}

This study was approved by our Ethics Committee in Shengjing Hospital, China Medical University (Shenyang, China) and each patient provided informed consent for the collection of tissue samples during surgery.

\section{Patient consent for publication}

Not applicable.

\section{Competing interests}

The authors declare that they have no competing interests.

\section{References}

1. Manchanda R and Menon U: Setting the threshold for surgical prevention in women at increased risk of ovarian cancer. Int J Gynecol Cancer 28: 34-42, 2018.

2. Oza AM, Cook AD, Pfisterer J, Embleton A, Ledermann JA, Pujade-Lauraine E, Kristensen G, Carey MS, Beale P, Cervantes A, et al: Standard chemotherapy with or without bevacizumab for women with newly diagnosed ovarian cancer (ICON7): Overall survival results of a phase 3 randomised trial. Lancet Oncol 16: 928-936, 2015.

3. Prat J: Ovarian carcinomas: Five distinct diseases with different origins, genetic alterations, and clinicopathological features. Virchows Arch 460: 237-249, 2012.

4. Siegel RL, Miller KD and Jemal A: Cancer statistics, 2017. CA Cancer J Clin 67: 7-30, 2017.

5. Schuijer M and Berns EM: TP53 and ovarian cancer. Hum Mutat 21: 285-291, 2003

6. Balch C, Fang F, Matei DE, Huang TH and Nephew KP: Minireview: Epigenetic changes in ovarian cancer. Endocrinology 150: 4003-4011, 2009.

7. Eddy SR: Non-coding RNA genes and the modern RNA world Nat Rev Genet 2: 919-929, 2001.

8. Wilusz JE, Sunwoo H and Spector DL: Long noncoding RNAs: Functional surprises from the RNA world. Genes Dev 23: 1494-1504, 2009.

9. Guttman M, Amit I, Garber M, French C, Lin MF, Feldser D, Huarte M,Zuk O, Carey BW, Cassady JP, et al: Chromatin signature reveals over a thousand highly conserved large non-coding RNAs in mammals. Nature 458: 223-227, 2009.

10. Mattick JS: The genetic signatures of noncoding RNAs. PLoS Genet 5: e1000459, 2009.

11. Geretto M, Pulliero A, Rosano C, Zhabayeva D, Bersimbaev R and Izzotti A: Resistance to cancer chemotherapeutic drugs is determined by pivotal microRNA regulators. Am J Cancer Res 7: 1350-1371, 2017.
12. Lu YM, Shang C, Ou YL, Yin D, Li YN, Li X, Wang N and Zhang SL: miR-200c modulates ovarian cancer cell metastasis potential by targeting zinc finger E-box-binding homeobox 2 (ZEB2) expression. Med Oncol 31: 134, 2014.

13. Guttman M and Rinn JL: Modular regulatory principles of large non-coding RNAs. Nature 482: 339-346, 2012.

14. Wapinski $\mathrm{O}$ and Chang HY: Long noncoding RNAs and human disease. Trends Cell Biol 21: 354-361, 2011.

15. Gutschner T and Diederichs S: The hallmarks of cancer: A long non-coding RNA point of view. RNA Biol 9: 703-719, 2012.

16. Gibb EA, Vucic EA, Enfield KS, Stewart GL, Lonergan KM, Kennett JY, Becker-Santos DD, MacAulay CE, Lam S, Brown CJ and Lam WL: Human cancer long non-coding RNA transcriptomes. PLoS One 6: e25915, 2011.

17. Gupta RA, Shah N, Wang KC, Kim J, Horlings HM, Wong DJ, Tsai MC, Hung T, Argani P, Rinn JL, et al: Long non-coding RNA HOTAIR reprograms chromatin state to promote cancer metastasis. Nature 464: 1071-1076, 2010.

18. Yang Z, Zhou L, Wu LM, Lai MC, Xie HY, Zhang F and Zheng SS: Overexpression of long non-coding RNA HOTAIR predicts tumor recurrence in hepatocellular carcinoma patients following liver transplantation. Ann Surg Oncol 18: 1243-1250, 2011.

19. Qiu JJ, Lin YY, Ye LC, Ding JX, Feng WW, Jin HY, Zhang Y, Li Q and Hua KQ: Overexpression of long non-coding RNA HOTAIR predicts poor patient prognosis and promotes tumor metastasis in epithelial ovarian cancer. Gynecol Oncol 134: 121-128, 2014.

20. Kogo R, Shimamura T, Mimori K, Kawahara K, Imoto S, Sudo T, Tanaka F, Shibata K, Suzuki A, Komune S, et al: Long noncoding RNA HOTAIR regulates polycomb-dependent chromatin modification and is associated with poor prognosis in colorectal cancers. Cancer Res 71: 6320-6326, 2011.

21. Szafron LM, Balcerak A, Grzybowska EA, Pienkowska-Grela B, Podgorska A, Zub R, Olbryt M, Pamula-Pilat J, Lisowska KM, Grzybowska E, et al: The putative oncogene, CRNDE, is a negative prognostic factor in ovarian cancer patients. Oncotarget 6: 43897-43910, 2015.

22. Barber HR, Sommers SC, Synder R and Kwon TH: Histologic and nuclear grading and stromal reactions as indices for prognosis in ovarian cancer. Am J Obstet Gynecol 121: 795-807, 1975.

23. Shannon P, Markiel A, Ozier O, Baliga NS, Wang JT, Ramage D, Amin N, Schwikowski B and Ideker T: Cytoscape: A software environment for integrated models of biomolecular interaction networks. Genome Res 13: 2498-2504, 2003.

24. Livak KJ and Schmittgen TD: Analysis of relative gene expression data using real-time quantitative PCR and the 2(-Delta Delta C(T)) method. Methods 25: 402-408, 2001.

25. Rinn JL, Kertesz M, Wang JK, Squazzo SL, Xu X, Brugmann SA, Goodnough LH, Helms JA, Farnham PJ, Segal E and Chang HY: Functional demarcation of active and silent chromatin domains in human HOX loci by noncoding RNAs. Cell 129: 1311-1323, 2007.

26. Qin LX, Beyer RP, Hudson FN, Linford NJ, Morris DE and Kerr KF: Evaluation of methods for oligonucleotide array data via quantitative real-time PCR. BMC Bioinformatics 7: 23, 2006.

27. Bhan A, Soleimani M and Mandal SS: Long noncoding RNA and cancer: A new paradigm. Cancer Res 77: 3965-3981, 2017.

28. Chen S, Wu DD, Sang XB, Wang LL, Zong ZH, Sun KX, Liu BL and Zhao Y: The lncRNA HULC functions as an oncogene by targeting ATG7 and ITGB1 in epithelial ovarian carcinoma. Cell Death Dis 8: e3118, 2017.

29. Fu X, Zhang L, Dan L, Wang K and Xu Y: LncRNA EWSAT1 promotes ovarian cancer progression through targeting miR-330-5p expression. Am J Transl Res 9: 4094-4103, 2017.

30. Yu J, Han Q and Cui Y: Decreased long non-coding RNA SPRY4-IT1 contributes to ovarian cancer cell metastasis partly via affecting epithelial-mesenchymal transition. Tumour Biol 39: $1010428317709129,2017$.

31. Zhu FF, Zheng FY, Wang HO, Zheng JJ and Zhang Q: Downregulation of lncRNA TUBA4B is associated with poor prognosis for epithelial ovarian cancer. Pathol Oncol Res 24: 419-425, 2018

32. Guo L, Peng Y, Meng Y, Liu Y, Yang S, Jin H and Li Q: Expression profiles analysis reveals an integrated miRNA-lncRNA signature to predict survival in ovarian cancer patients with wild-type BRCA1/2. Oncotarget 8: 68483-68492, 2017.

33. Wang L, Hu Y, Xiang X, Qu K and Teng Y: Identification of long non-coding RNA signature for paclitaxel-resistant patients with advanced ovarian cancer. Oncotarget 8: 64191-64202, 2017. 
34. Cao Y, Shi H, Ren F, Jia Y and Zhang R: Long non-coding RNA CCAT1 promotes metastasis and poor prognosis in epithelial ovarian cancer. Exp Cell Res 359: 185-194, 2017.

35. Shen L, Liu W, Cui J, Li J and Li C: Analysis of long non-coding RNA expression profiles in ovarian cancer. Oncol Lett 14: 1526-1530, 2017

36. Ding Y, Yang DZ, Zhai YN, Xue K, Xu F, Gu XY and Wang SM: Microarray expression profiling of long non-coding RNAs in epithelial ovarian cancer. Oncol Lett 14: 2523-2530, 2017.

37. Kaur P, Tan JR, Karolina DS, Sepramaniam S, Armugam A, Wong PT and Jeyaseelan K: A long non-coding RNA, BC048612 and a microRNA, miR-203 coordinate the gene expression of neuronal growth regulator 1 (NEGR1) adhesion protein. Biochim Biophys Acta 1863: 533-543, 2016.
38. Ji J, Tang J, Deng L, Xie Y, Jiang R, Li G and Sun B: LINC00152 promotes proliferation in hepatocellular carcinoma by targeting EpCAM via the mTOR signaling pathway. Oncotarget 6 : 42813-42824, 2015.

39. Chen H, Du G, Song $X$ and Li L: Non-coding transcripts from enhancers: New insights into enhancer activity and gene expression regulation. Genomics Proteomics Bioinformatics 15: 201-207, 2017. 